\title{
Comparison of proper motions in declination for 387 Gaia DR2 and HIPPARcos stars from ILS observations over many decades ${ }^{\star}$
}

\author{
G. Damljanović ${ }^{1}$ and F. Taris ${ }^{2}$
}

\author{
1 Astronomical Observatory, Volgina 7, 11060 Belgrade, Serbia \\ e-mail: gdamljanovic@aob.rs \\ 2 Observatoire de Paris - SYRTE, PSL Research University, CNRS/UMR 8630, Sorbonne Universités, Université Pierre et Marie \\ Curie, LNE, 61 avenue de l'Observatoire, 75014 Paris, France \\ e-mail: Francois.Taris@obspm. fr
}

Received 21 August 2019 / Accepted 26 September 2019

\begin{abstract}
Context. The second solution of the Gaia catalog, which has been available since April 2018, plays an important role in the realization of the future Gaia reference frame. Since 1997, the reference frame has been materialized by the optical HIPPARCOS positions of about 120000 stars. The HIPPARCos has been compared with and linked to the International Celestial Reference Frame (ICRF). The ICRF is materialized by means of the radio positions of extragalactic sources using very large baseline interferometry observations. Both, the HIPPARCos and Gaia missions belong to the European Space Agency, and it is important to note that the Gaia catalog is going to replace the HIPPARCos catalog.

Aims. It has been shown that the International Latitude Service zenith telescope data pertaining to ground-based surveys that span a time baseline of about $80 \mathrm{yr}$, and which are also key when measuring proper motions, could be useful for the accurate determination of $\mu_{\delta}$ for 387 ILS stars. Therefore, in this study we aim first to reduce these stars to the HIPPARCos reference system; second, to made our original catalog of $\mu_{\delta}$, which we refer to as the ILS catalog, for these 387 bright stars; third, to present comparison results of the four catalogs by pairs (the ILS, HIPPARCOS or HIP, new HIPPARCOS or NHIP, and Gaia DR2); and fourth, to analyze the differences in $\mu_{\delta}$ between pairs of catalogs to characterize the $\mu_{\delta}$ errors for these catalogs with a special focus on the Gaia DR2 and ILS catalogs. Methods. At seven ILS sites around the world at latitude 39.1, a set of seven telescopes was used to monitor the latitude variation via observations of the same stars for about $80 \mathrm{yr}$. Here, the inverse task was applied to improve $\mu_{\delta}$ values of the 387 HIPPARCOS stars using the previously mentioned observations. Due to the specific Horrebow-Talcott method of the measured star pair, it is difficult to determine $\mu_{\delta}$ for each single star. However, we achieved this by developing the original method and in combination with the HIPPARCos data. We used the previously developed least squares method and formula to determine the coefficients, which describe the systematic part of differences in $\mu_{\delta}$ between the pairs of catalogs.

Results. We calculated the coefficients with the aforementioned formula (in line with the coordinates, stellar magnitude, and color index of every star) to compare ILS, HIP, NHIP, and Gaia DR2 data of $\mu_{\delta}$ against each other by using the set of 387 stars. The presented differences of $\mu_{\delta}$ show that the systematic errors in the four catalogs are nearly at the same level of $0.1 \mathrm{mas}^{-1}$. This means that the DR2 and ILS $\mu_{\delta}$ values are in good agreement with each other, and with values from the HIPPARCos and new HIPPARCos catalogs. Also, the random errors of differences are small ones; they are near 1 mas $\mathrm{yr}^{-1}$ for ILS-HIP and ILS-NHIP, and about $2 \mathrm{mas} \mathrm{yr}^{-1}$ for ILS-DR2, HIP-DR2, and NHIP-DR2. It is important to note that there is a similar level of proper motion formal errors in HIPPARCOS and new HIPPARCos catalogs.
\end{abstract}

Key words. astrometry - catalogs - proper motions

\section{Introduction}

Gaia is a mission of the European Space Agency (ESA). It started its five-year nominal operation phase in July 2014, nearly six months after launching the Gaia satellite in December 2013 (Gaia Collaboration 2016a). The goal was to collect astronomical data (positions, proper motions, and parallaxes) for more than one billion sources brighter than $20.7 \mathrm{mag}$ in $G$-band, and for about 500000 quasars (Prusti 2012). There have been two releases so far, Gaia DR1 in September 2016 and DR2 in April 2018 (Gaia Collaboration 2016b; Lindegren et al. 2018). The Gaia DR1 results are based on observations collected during the

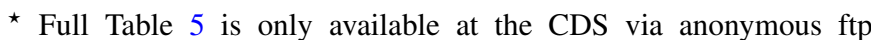
to cdsarc.u-strasbg. fr (130.79.128.5) or via http://cdsarc. u-strasbg.fr/viz-bin/cat/J/A+A/631/A145 first 14 months starting with July 2014, and DR2 result are from the first 22 months.

Here, we used the HIPPARCos catalog data (ESA 1997) and the new HIPPARCos catalog data (van Leeuwen 2007) to compare the $\mu_{\delta}$ values against those from Gaia DR2 and ILS. The ILS is our original catalog of $\mu_{\delta}$ for 387 ILS stars (Damljanović \& Pejović 2006). The epoch of the HIPPARCos catalog is J1991.25, and it was the first satellite catalog with about 120000 stars. It was an ESA mission as well, and that catalog appeared in 1997 as a new reference frame (the ICRS in the optical domain).

To obtain the original ILS catalog of $\mu_{\delta}$ values for 387 stars and to improve the $\mu_{\delta \mathrm{H}}$ from the HIPPARCos catalog, we took into account the latitude variations made using instruments at seven sites (see Table 1) during the period of 1899.7-1979.0. We note that approximately an 80-year-long period of ILS data is necessary to get accurate $\mu_{\delta}$ values. The ILS data were included 
Table 1. ILS stations, their geographic coordinates (longitude $\lambda_{\mathrm{W}}$ and mean latitude $\varphi$ ), and observed intervals.

\begin{tabular}{|c|c|}
\hline $\begin{array}{l}\text { ILS station } \\
\lambda_{\mathrm{W}}\left(^{\circ}\right), \varphi\left(39^{\circ} 8^{\prime}+{ }^{\prime \prime}\right)\end{array}$ & $\begin{array}{l}\text { Observed } \\
\text { interval }\end{array}$ \\
\hline $\begin{array}{l}\text { 1. Carloforte (CA) } \\
351.7,9.157\end{array}$ & $\begin{array}{l}\text { 1899.8-1979.0 } \\
\text { (break: 1943.3-1946.5) }\end{array}$ \\
\hline $\begin{array}{l}\text { 2. Cincinnati (CI) } \\
84.4,19.437\end{array}$ & $1899.8-1916.0$ \\
\hline $\begin{array}{l}\text { 3. Gaithersburg (GT) } \\
77.2,13.309\end{array}$ & $\begin{array}{l}\text { 1899.8-1979.0 } \\
\text { (break: 1915.0-1932.6) }\end{array}$ \\
\hline $\begin{array}{l}\text { 4. Kitab (KZ) } \\
293.1,2.057 \\
\end{array}$ & $1930.8-1979.0$ \\
\hline $\begin{array}{l}\text { 5. Mizusawa (MZZ) } \\
218.9,3.687\end{array}$ & $1900.0-1979.0$ \\
\hline $\begin{array}{l}\text { 6. Tschardjui (TS) } \\
296.5,11.334\end{array}$ & 1899.7-1919.3 \\
\hline $\begin{array}{l}\text { 7. Ukiah (UK) } \\
123.2,12.161\end{array}$ & 1899.7-1961.0 \\
\hline
\end{tabular}

as input data into the Bureau International de l'Heure (BIH) in Paris as part of the Earth rotation programs to produce the Earth orientation parameters (EOP).

In the following, we present information about $\mu_{\delta}$ data from the ILS and the other previously mentioned catalogs. In the next section, the calculation steps necessary to obtain the proper motion in declination for 387 ILS stars are performed. The error analysis, for random and systematic errors, regarding the comparison of $\mu_{\delta}$ between the four catalogs is found in Sect. 4. The Conclusion details the main results after comparing the four catalogs by pairs, and recommendations are given about useful ground-based data in the framework of the satellite Gaia mission.

\section{ILS, Hipparcos, and Gaia DR2 data}

Gaia DR2 contains results for about 1.69 billion sources in the $G$ magnitude range from 3 to 21 and at the reference epoch J2015.5. There are about 1.33 billion sources with five astrometric parameters (coordinates, proper motions, and parallaxes) and the approximate positions for 0.36 billion faint objects. For 1.33 billion sources, the median uncertainty in position and parallax is about 0.04 mas for sources with $G<14$ mag at J2015.5,

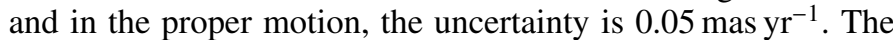
Gaia DR2 optical reference frame is aligned with the ICRS via quasars (Lindegren et al. 2018). Due to calibration issues, the stars with $G<6$ mag mostly have inferior astrometry; here, about one third of ILS stars are in that group (see Fig. 2). The DR1 solution (or Tycho-Gaia astrometric solution) incorporated astrometric HIPPARCOS and Tycho-2 data (Lindegren et al. 2016), but the DR2 solution is independent of HIPPARCOS and Tycho-2 catalogs. In both solutions (DR1 and DR2), the sources are treated as single stars; for unresolved binaries the presented data thus refer to the photocenter. For resolved binaries, the results are sometimes spurious due to the confusion of the components.

The HIPPARcos catalog is the optical counterpart (Kovalevsky et al. 1997) of the ICRF. The HIPPARCos stars (118218 ones) are brighter than $V$-mag 12. Mostly, they are between $7 \mathrm{mag}$ and $9 \mathrm{mag}$. The errors in position and parallax are about 1 mas at J1991.25, and in the proper motion about

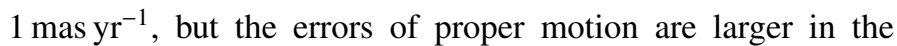
case of double stars than for single ones. The main reason is due to the observation period, which was less than four years (Vondrák et al. 1998). The new HIPPARcos catalog has appeared to improve the coordinates, proper motions, and parallaxes of stars. (van Leeuwen 2007) performed a new reduction of raw HIPPARCOS observations, and the new astrometric data are better by a factor of 2.2 in total weight, and by up to a factor of four for almost all stars that are brighter than 8 mag. All ILS stars here are brighter than 8 mag (see Fig. 2). Finally, it is possible to get more accurate values, pertaining to positions and proper motions, by combining the ground-based and satellite data (such as HIPPARCos data) with those from the HIPPARCos catalog. This is the case for ARIHIP, the Earth Orientation Catalog (EOC; Vondrák et al. 2003), and also other catalogs.

There have been many astrometric ground-based observations of the same stars, which are found in ILS data, referred to the HIPPARCos catalog, and made using many instruments during the last century. In the case of ILS data, 387 HIPPARCos stars were observed at seven stations (Carloforte, Cincinnati, Gaithersburg, Kitab, Mizusawa, Tschardjui, and Ukiah), covering the period from 1899.7-1979.0 (see Table 1). Our ILS catalog of $\mu_{\delta}$ (Damljanović \& Pejović 2006) yields accurate data for 387 stars that are common to ILS and HIPPARCos. To get precise $\mu_{\delta}$ via ILS data, we took a reverse approach by using latitude observations and combined these ground-based data with satellite HIPPARCos data. The original method was applied (Damljanović 2007); the corrections $\Delta \mu_{\delta}$ of suitable HIPPARCOS $\mu_{\delta_{\mathrm{H}}}$ were calculated and applied to the corresponding $\mu_{\delta_{\mathrm{H}}}$ values. Finally, the ILS values $\mu_{\delta}$ for 387 stars show a high accuracy and are in good agreement with Gaia DR2, HIPPARCOS, and new HIPPARCOS values.

\section{Calculation of proper motion in declination of $\mathbf{3 8 7}$ ILS stars using latitude observations}

\subsection{ILS latitude observations}

The error of $\mu_{\delta}$ is in line with $1 / \Delta t$. Because of it, using a long ILS time interval of about $80 \mathrm{yr}$ makes it possible to get a better accuracy of $\mu_{\delta}$ (for 387 ILS stars) than for the HIPPARCOS stars. Even the accuracy of HIPPARCos star positions are better than those from ground-based surveys. Also, there are many observations of the same ILS star pair (from a few to a few hundred times) per each year for the period from 1899.7 to 1979.0. Even about 0 '!2 of zenith-telescope accuracy, the long time interval (from a few decades to potentially about $80 \mathrm{yr}$ ), and numerous observations per year are of importance for better accuracy of ILS $\mu_{\delta}$ values than for the HIPPARCos values. Plus, by combining the ILS observations with suitable HIPPARCOS data for the common stars, we can get better results than if only groundbased data are used. The point of HIPPARCos data is done for the epoch 1991.25, and during calculation we used suitable weights for all points, which were inversely proportional to position errors (Damljanović et al. 2006). The tectonic plate motion and the mean latitude were removed from the ILS observations (Vondrák, priv. comm.); more information about ILS can be found in the publication by Yumi \& Yokoyama (1980).

In accordance with the Horrebow-Tallcott method, the main zenith-telescope (ZT) formula to calculate latitude $\varphi_{\mathrm{P}}$ from a star pair and for the moment of measurement $t$ is $\varphi_{\mathrm{P}}=\left(\delta_{\mathrm{S}}-\delta_{\mathrm{N}}\right) / 2+$ $\Delta z / 2$. The values $\delta_{\mathrm{S}}$ and $\delta_{\mathrm{N}}$ are the apparent declinations of stars (south star and north star, respectively) in the star pair, and the zenith-distance difference $\Delta z=z_{\mathrm{S}}-z_{\mathrm{N}}$ is from ZT observations. 


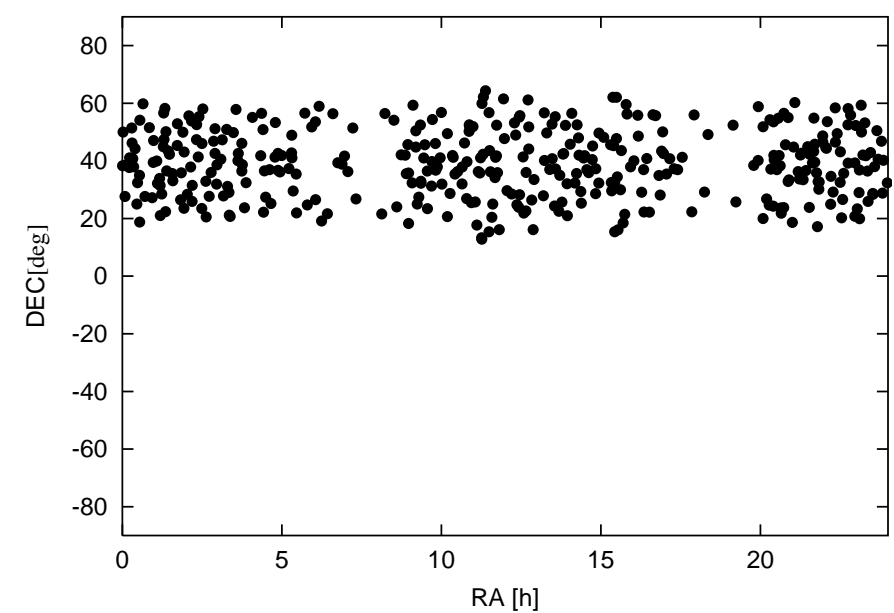

Fig. 1. Distribution of 387 stars on celestial sphere.

Values $\delta_{\mathrm{S}}$ and $\delta_{\mathrm{N}}$ were calculated by using the HIPPARCos catalog. If we remove the polar motion term and systematic errors (instrumental, local, etc.) from the latitude data, the residuals are catalog errors that is of interest to us (Damljanović et al. 2006).

\subsection{Proper motions in declination using latitude observations}

In line with $\Delta \varphi_{\mathrm{P}}+\left(\mathrm{d} \varphi_{\mathrm{P}} / \mathrm{d} t\right) t \approx\left(\Delta \delta_{\mathrm{S}}+\Delta \delta_{\mathrm{N}}\right) / 2+t\left(\Delta \mu_{\delta_{\mathrm{S}}}+\Delta \mu_{\delta_{\mathrm{N}}}\right) / 2$ (Vondrák et al. 1998), we can obtain $a$ and $b$ by using the least square method (LSM) and linear model $\operatorname{res}_{n}=a+b\left(t_{n}-1991.25\right)$ on input points with weights. The values $\Delta \delta_{\mathrm{S}}$ and $\Delta \delta_{\mathrm{N}}$ are corrections of declinations, $\Delta \mu_{\delta_{\mathrm{S}}}$ and $\Delta \mu_{\delta_{\mathrm{N}}}$ are corrections of proper motions in declination, $t$ is time, res $_{n}$ is the star pair residual, $t_{n}$ (in years) is the epoch of $\operatorname{res}_{n}, a$ pertains to $\left(\Delta \delta_{\mathrm{S}}+\Delta \delta_{\mathrm{N}}\right) / 2$, and $b$ pertains to $\left(\Delta \mu_{\delta_{\mathrm{S}}}+\Delta \mu_{\delta_{\mathrm{N}}}\right) / 2$. The residuals of latitude variations res $_{n}$ are without polar motion and the systematic terms (Damljanović 2005), $a$ and $b$ are in accordance with the epoch 1991.25.

There is one equation $b=\left(\Delta \mu_{\delta_{\mathrm{S}}}+\Delta \mu_{\delta_{\mathrm{N}}}\right) / 2$, but two unknowns $\Delta \mu_{\delta_{\mathrm{S}}}$ and $\Delta \mu_{\delta_{\mathrm{N}}}$. To solve that problem, we introduced one more Eq. (1), as part of our original procedure,

$\Delta \mu_{\delta_{\mathrm{S}}}-\Delta \mu_{\delta_{\mathrm{N}}}=\left(\mu_{\delta_{\mathrm{S} 1}}-\mu_{\delta_{\mathrm{S} 2}}\right)-\left(\mu_{\delta_{\mathrm{N} 1}}-\mu_{\delta_{\mathrm{N} 2}}\right)$,

where $\mu_{\delta_{\mathrm{S} 1}}$ and $\mu_{\delta_{\mathrm{N} 1}}$ are from the EOC-2 catalog (Vondrák 2004), and $\mu_{\delta_{\mathrm{S} 2}}$ and $\mu_{\delta_{\mathrm{N} 2}}$ are from the HIPPARCos catalog. About the errors, $\epsilon \Delta_{\mu_{\delta_{\mathrm{S}}}}$ and $\epsilon \Delta_{\mu_{\delta_{\mathrm{N}}}}$ are standard deviation of $\Delta \mu_{\delta_{\mathrm{S}}}$ and $\Delta \mu_{\delta_{\mathrm{N}}}$, respectively. There is one equation $\left(\epsilon^{2} \Delta_{\mu_{\delta_{\mathrm{S}}}}+\epsilon^{2} \Delta_{\mu_{\delta_{\mathrm{N}}}}\right) / 2=\epsilon_{\mathrm{b}}^{2}$ but two unknowns $\epsilon \Delta_{\mu_{\delta \mathrm{S}}}$ and $\epsilon \Delta_{\mu_{\delta_{\mathrm{N}}}}$. To solve that problem, we performed one more Eq. (2):

$\epsilon \Delta_{\mu_{\delta_{\mathrm{S}}}} / \epsilon \Delta_{\mu_{\delta_{\mathrm{N}}}}=\epsilon \Delta_{\mu_{\delta_{\mathrm{S} 1}}} / \epsilon \Delta_{\mu_{\delta_{\mathrm{N} 1}}}$,

where $\epsilon \Delta_{\mu_{\delta_{S 1}}}$ and $\epsilon \Delta_{\mu_{\delta_{\mathrm{N} 1}}}$ are from EOC-2; $\epsilon \Delta_{\mu_{\delta_{\mathrm{S} 1}}}$ and $\epsilon \Delta_{\mu_{\delta_{\mathrm{N} 1}}}$ are the errors of stars $S$ (south star) and $N$ (north star) of the ILS star pair, respectively. In that direction, it is possible to calculate corrections $\Delta \mu_{\delta_{\mathrm{S}}}$ and $\Delta \mu_{\delta_{\mathrm{N}}}$ (with their errors) of each star pair for common ILS and HIPPARCOS stars, to apply these corrections to corresponding HIPPARCOS values $\mu_{\delta_{\mathrm{H}}}$, and to obtain the ILS catalog with proper motions in declination for each star separately. After which, it is of interest to compare these $\mu_{\delta}$ values of 387 stars with Gaia DR2, HIPPARCos, and new HIPPARCos values. This is especially the case for Gaia DR2 because the ILS catalog depends of HIPPARCOS and EOC-2 data.

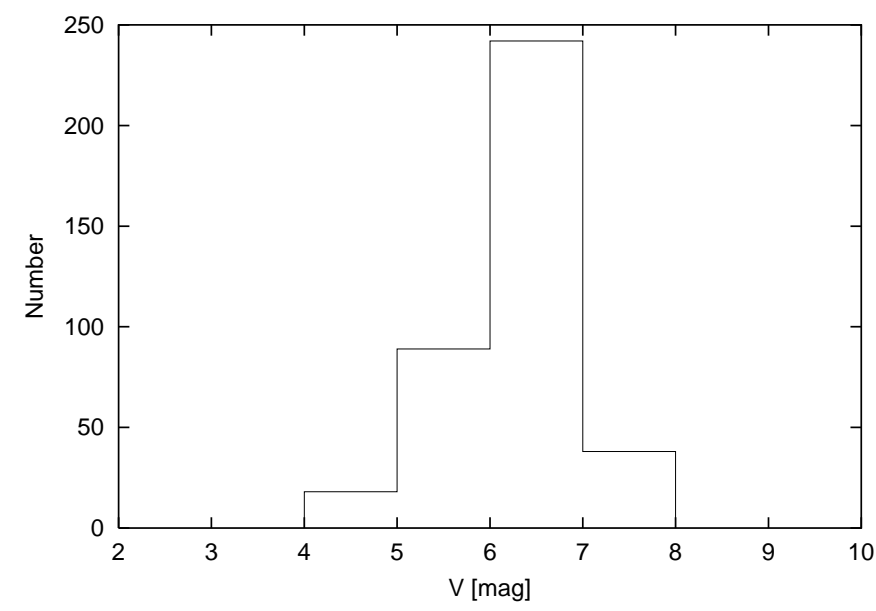

Fig. 2. Distribution of magnitudes of 387 stars.

\section{Results}

In Fig. 1, the distribution of 387 stars on the celestial sphere is presented. The values of $0^{\mathrm{h}}<\alpha<24^{\mathrm{h}}$, but $20^{\circ}<\delta<60^{\circ}$; it is possible to investigate the systematics (via the comparison of four catalogs of pairs by using suitable differences of $\mu_{\delta}$ ) over all $\alpha$ (see Fig. 3), but over just a part of $\delta$ (see Fig. 4). The distribution of $V$ magnitude is presented in Fig. $2(4<V<8$, mostly ILS stars are from 6 to $7 \mathrm{mag}$ ), and the differences as function of $V$ magnitude are presented in Fig. 5. Suitable mean proper motion differences (as a function of $\alpha, \delta$, and $V$ magnitude) are presented in Figs. 3-5.

The average values of $\epsilon_{\mu_{\delta}}\left(\mathrm{mas} \mathrm{yr}^{-1}\right)$ of 387 stars for the ILS catalog, which are also in line with $V$ magnitude, are in Table 2. That value in the case of all HIPPARCos stars is

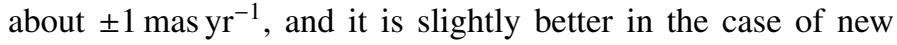
HIPPARCOS.

Figures 3-5 indicate some systematic errors between the mean data values from the catalog pairs. Differences between ILS and DR2 are somewhat higher in the central part of Fig. 3 (at $12^{\mathrm{h}}$ of $\alpha$ ), and there is a small sinusoidal curve with an ampli-

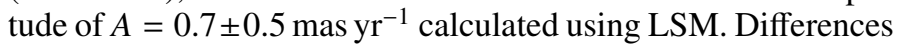
presented in Fig. 4 are mostly near zero; also, $\delta$ is just in an interval from $20^{\circ}$ to $60^{\circ}$. In Fig. 5, only for stars fainter than 7 mag in the $V$ band are the differences slightly higher than for the other stars. This means that the ILS values of $\mu_{\delta}$ are in good agreement with DR2, HIPPARCos, and new HIPPARCos data. In Figs. 3-5,

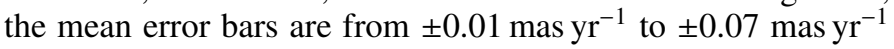
for the plotted mean differences.

The $\mu_{\delta}$ differences (or $\Delta$ ) on $\alpha, \delta$, and $V$ magnitude (in Figs. 3-5) are small, but we tried to calculate random and systematic errors of pairwise differences of 387 common stars from the catalogs using the following formula (Ivanov \& Yatsenko 2003):

$k_{1}+k_{2}\left(V-V_{0}\right)+k_{3}(B-V)=\Delta$,

where $V_{0}=6.28$ is the mean value of $V$ magnitudes and $(B-V)$ is the color index of a star. The unknowns $k_{1}, k_{2}$, and $k_{3}$ are determined using LSM and presented in Table 3; they describe the systematic part of $\mu_{\delta}$ differences. Also, in Table 3 the sum $s_{0}$ of the random errors for both catalogs is found. Meaning, $s_{0}$ is the random part of $\Delta$ (the unit weight error of the solution of the system). It is calculated as $s_{0}=$ $\left\{\frac{1}{387-3} \sum_{n=1}^{387}\left[\Delta_{n}-\left(k_{1}+k_{2}\left(V_{n}-V_{0}\right)+k_{3}(B-V)_{n}\right)\right]^{2}\right\}^{1 / 2}$ where $1 \leq$ $n \leq 387$. 


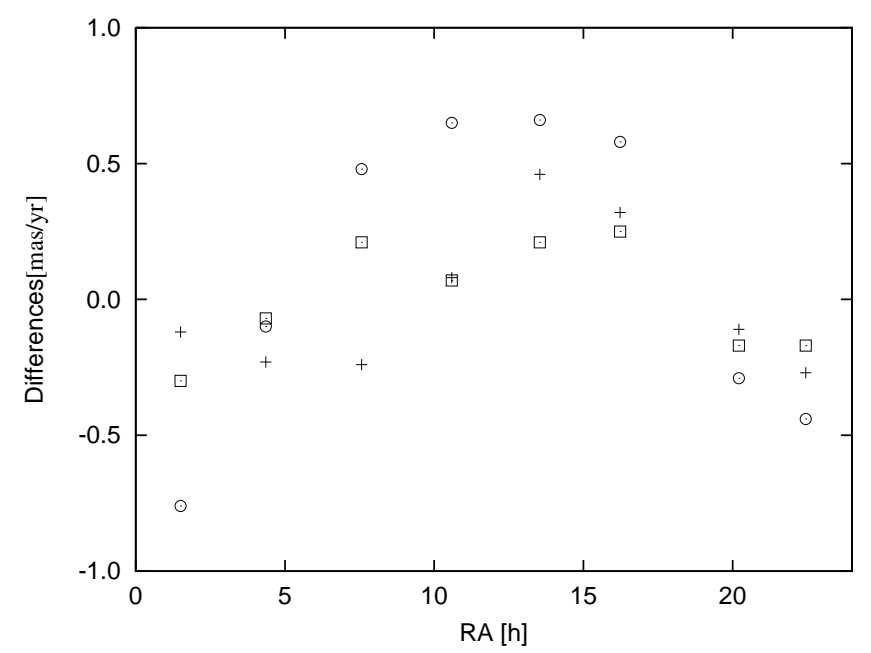

Fig. 3. Mean proper motion differences as function of $\alpha$ : ILS-HIP (cross), ILS-NHIP (open rectangle), ILS-DR2 (open circle).

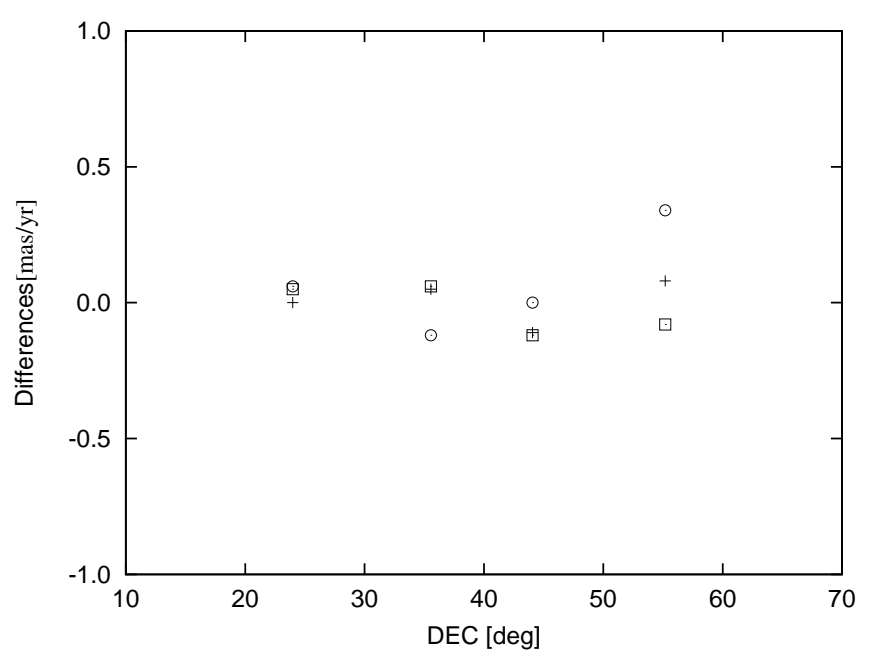

Fig. 4. Mean proper motion differences as function of $\delta$; designations are the same as in Fig. 3.

In the ILS and HIP as well as the ILS and NHIP cases, the value $s_{0} \approx 1 \mathrm{mas} \mathrm{yr}^{-1}$. However, in the case of ILS and DR2, it

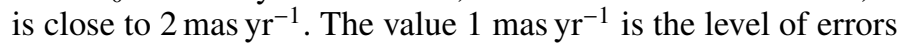
of all HIPPARCos $\mu_{\delta}$ data. As previously mentioned, Gaia DR2 stars with $G<6$ mag have inferior astrometry (Lindegren et al. 2016), and this could be the reason for $s_{0} \approx 2 \mathrm{mas} \mathrm{yr}^{-1}$ in combination for ILS and DR2. The coefficients $k_{1}, k_{2}$, and $k_{3}$ (in Table 3) are small for any combination of catalogs, and their standard errors are higher than the corresponding values. As we can see, the random and systematic errors of $\Delta$ are small and mostly close to each other. The values $\mu_{\delta}$ of four catalogs are with high accuracy and no significant relationship between $\Delta$ and $\left(V-V_{0}\right)$ (also, $\left.(B-V)\right)$ exists in any pair of catalogs. Using 387 stars here, the values $\mu_{\delta}$ of Gaia DR2, which are independent and based on 22 months of Gaia observations, are in good accordance with ILS data.

There are 18 double and multiple stars in the ILS catalog (see Table 4); some information is from HIPPARcos and the Sixth Catalog of Orbits of Visual Binary Stars. In three cases, the orbital period $P<100 \mathrm{yr}$, and the orbital motion of double and multiple stars, can influence proper motions. This depends on $P$. The short period of HIPPARCos observations is negligible

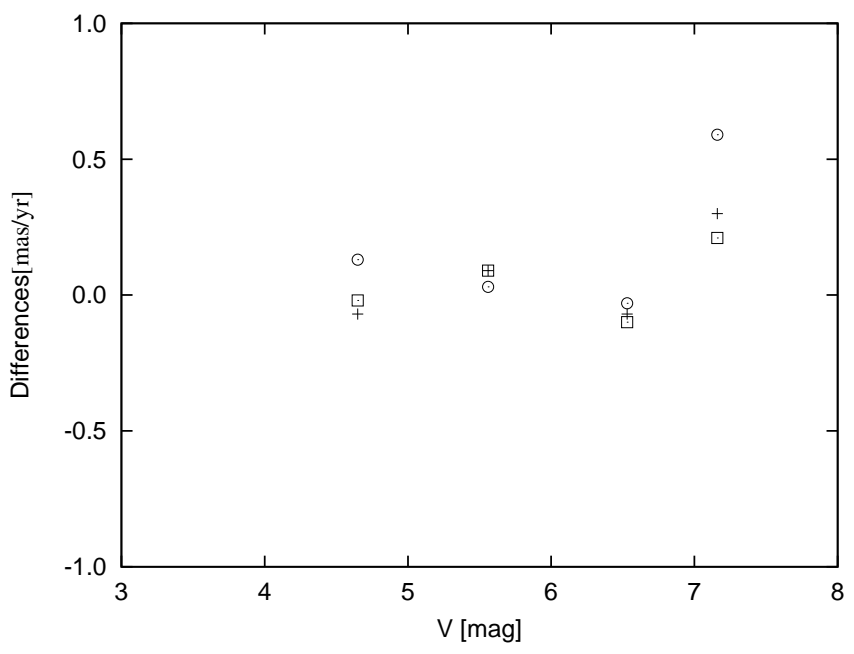

Fig. 5. Mean proper motion differences as function of magnitude; designations are the same as in Fig. 3.

Table 2. Average value of $\epsilon_{\mu \delta}$ of catalog ILS using 387 stars.

\begin{tabular}{ll}
\hline \hline Average value of $\epsilon_{\mu \delta}$ & \\
& {$\left[\mathrm{mas} \mathrm{yr}^{-1}\right.$ ] } \\
\hline ILS, 387 stars & \pm 0.21 \\
$V$ mag from 4 to 5 & \pm 0.17 \\
Vmag from 5 to 6 & \pm 0.21 \\
Vmag from 6 to 7 & \pm 0.21 \\
Vmag from 7 to 8 & \pm 0.18 \\
\hline
\end{tabular}

Table 3. Comparison of $387 \mu_{\delta}$ values from catalogs ILS, HIP, NHIP, and Gaia DR2 to calculate formal and systematic errors.

\begin{tabular}{ll}
\hline \hline Catalog,$s_{0}\left[\mathrm{mas} \mathrm{yr}^{-1}\right]$ & $\begin{array}{l}k_{1}, k_{2}, k_{3} \\
{\left[\mathrm{mas} \mathrm{yr}^{-1}\right]}\end{array}$ \\
\hline ILS-HIP , 1.11 & $-0.03,+0.04,+0.04$ \\
& $\pm 0.09 \pm 0.09 \pm 0.10$ \\
\hline ILS-NHIP , 1.16 & $-0.05,-0.02,+0.04$ \\
& $\pm 0.10 \pm 0.09 \pm 0.11$ \\
\hline ILS-DR2, 2.01 & $-0.05,+0.10,+0.14$ \\
& $\pm 0.17 \pm 0.16 \pm 0.18$ \\
\hline
\end{tabular}

in comparison with most of the $P$ values. It is not the case of ILS data because the observational period is almost $80 \mathrm{yr}$. Regarding the Gaia DR2, the published data refer to the photocenter for unresolved binaries. The ILS, HIPPARCOS, and Gaia DR2 observations are related to different positions on the orbital arc of some double or multiple stars. That time difference is more than a half century between the middle of the ILS and HIPPARCOS epochs; there is more than three quarters of a century between ILS and Gaia DR2 epochs. Here, it is of importance that $\mu_{\delta}$ values of ILS are obtained from many decades of observations. Nevertheless, some HIPPARCOS and Gaia DR2 proper motions data are not reliable even with small formal errors. This is due to many unresolved binaries, the ILS and other similar data with long histories could be useful to check even satellite data, such as HIPPARCos and Gaia data. Here, we detected two cases, H 55060 and H 117622, with big $\mu_{\delta}\left(\right.$ mas yr$\left.^{-1}\right)$ differences between ILS and DR2, which are as follows: 16.32 for H 55060, and 14.02 for H 117622, respectively. Subsequently in 
Table 4. Detected 18 double and multiple stars (in ILS catalog) with their HIPPARCOS/WDS number, comment, and period $P$ (day or yr).

\begin{tabular}{ll}
\hline \hline HIP/WDS & $P$ \\
\hline $10535 / 02157+2503$ & 8622.7 days \\
$15627 / 03212+2109$ eclipsing & \\
$15737 / 03228+2045$ no orbit & \\
$20586 / 04245+5051$ linear & \\
$22279 / 04478+5318$ & $309.9 \mathrm{yr}$ \\
$44064 / 08585+3548$ & $46.8 \mathrm{yr}$ \\
$54842 / 11137+4105$ no orbit & \\
$56274 / 11322+3615$ & $81.5 \mathrm{yr}$ \\
$56613 / 11365+2502$ no orbit & \\
$66086 / 13328+2421$ no orbit & \\
$73068 / 14560+3218$ no orbit & \\
$84606 / 17177+3717$ no orbit & \\
$100643 / 20244+2417$ no orbit & \\
$101214 / 20310+3656$ no orbit & \\
$108372 / 21573+6118$ no orbit & \\
$108845 / 22029+4439$ no orbit & \\
$112871 / 22514+2623$ yr & $383 \mathrm{yr}$ \\
$117340 / 23476+4650$ no orbit & \\
\hline
\end{tabular}

these cases, data of ILS, HIP, and NHIP are consistent between each other, but not with DR2. We did not find any explanation for this.

To add more information to the ILS catalog, we did a direct comparison between the NHIP and Gaia DR2 positions in declination (using an epoch difference of $24.25 \mathrm{yr}$ ) with the ILS $\mu_{\delta}$. It is $\mu_{\delta \mathrm{DR} 2 / \mathrm{NHIP}}=\left(\delta_{\mathrm{DR} 2}-\delta_{\mathrm{NHIP}}\right) / 24.25$ of a star. Two declinations are $\delta_{\mathrm{DR} 2}$ and $\delta_{\mathrm{NHIP}}$ of this star for the epochs $t_{\mathrm{DR} 2}=$ 2015.5 and $t_{\mathrm{NHIP}}=1991.25$, respectively. The error is $\epsilon_{\mu_{\delta}}=$ $\left(\epsilon_{\mathrm{DR} 2}^{2}+\epsilon_{\mathrm{NHIP}}^{2}\right)^{1 / 2} / 24.25$ (Eichhorn 1974); $\epsilon_{\mathrm{DR} 2}$ and $\epsilon_{\mathrm{NHIP}}$ are the standard errors of $\delta_{\mathrm{DR} 2}$ and $\delta_{\mathrm{NHIP}}$, respectively. The differences $\left(\mu_{\delta \mathrm{ILS}}-\mu_{\delta \mathrm{DR} 2 / \mathrm{NHIP}}\right)$, points, and main values over $3^{\mathrm{h}}$ subintervals of $\alpha$, are presented in Fig. 7. This is a different comparison as opposed to the one presented in Fig. 3, as it compares to the positional reference frames. There are three possible sources for the observed differences: a distortion in the HIPPARCOS positional reference frame, a distortion in the Gaia DR2 positional reference frame, or a distortion in the Gaia DR2 proper motions. Also, any combination of these is possible. Due to a small number of stars and limited accuracy, the ILS cannot provide a definitive answer about this. We detected one case involving substantial differences between $\mu_{\delta \mathrm{ILS}}$ and $\mu_{\delta \mathrm{DR} 2 / \mathrm{NHIP}}$ values for star H 62145 that is 13.266 mas $\mathrm{yr}^{-1}$. This is probably caused by

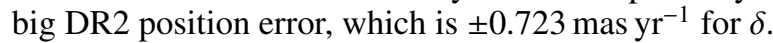

In Fig. 6, we show the distribution of formal uncertainties on

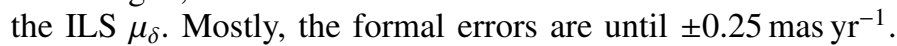
This is in line with NHIP and a suitable HIP value.

\section{Conclusion}

We have presented the original ILS catalog of $\mu_{\delta}$ data of 387 stars, and comparison results of the four catalogs by pairs (Gaia DR2, HIPPARCos, new HIPPARCos, and ILS) via $\mu_{\delta}$ of these stars. The ground-based observations of about an 80-year-long interval (during the period from 1899.7-1979.0) were done with a network of seven ILS instruments. These observations provide new information about $\mu_{\delta}$ of mentioned common ILS and HIPPARCOS stars. To obtain $\mu_{\delta}$ of 387 stars, the original method was applied and a combination of ILS, HIPPARCOS, and EOC-2

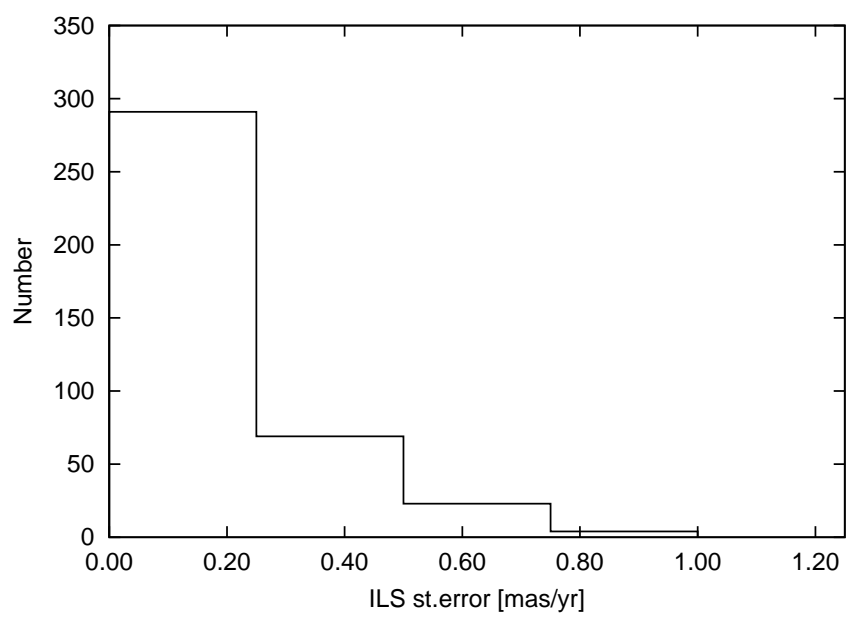

Fig. 6. Distribution of standard errors in $\mu_{\delta}$ of ILS catalog.

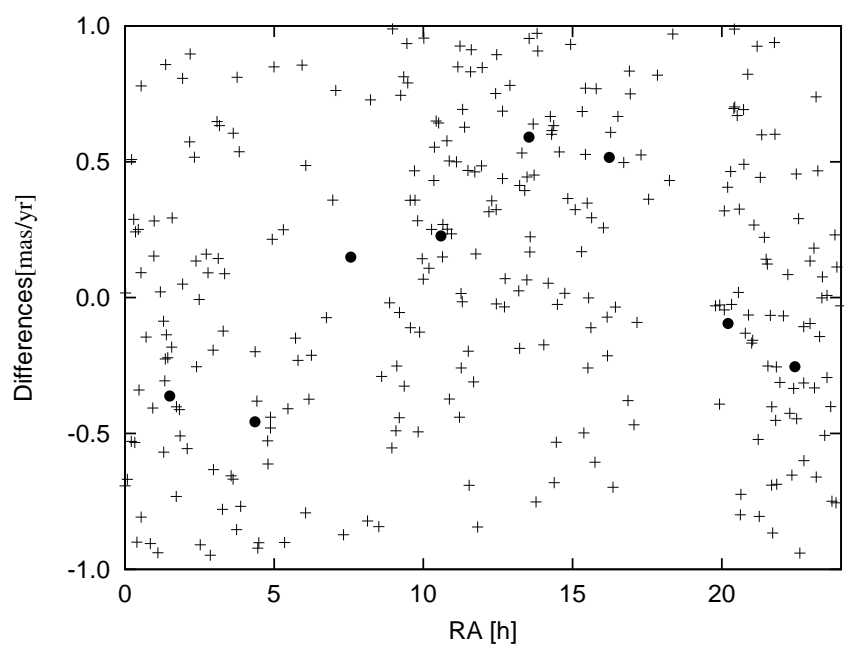

Fig. 7. Proper motion differences as function of $\alpha$, ILS-NHIP/DR2, points (cross), and mean values (circle).

data was used. The ILS catalog of $\mu_{\delta}$ satisfies the requirements of modern astrometry, and its advantage is the large number of observations together with long observation periods (several tens per star pair per year during several decades). The HIPPARCos period of observations is less than four years and 22 months in the case of Gaia DR2. The average value of $\epsilon_{\mu \delta}$ of ILS is between HIPPARCos, or new HIPPARCos, and Gaia DR2 ones values; the Gaia DR2 is the best catalog. After comparing the four catalogs in pairs, good consistency between these catalogs was found with no significant discrepancies. The analysis of random and systematic errors via the (Ivanov \& Yatsenko 2003) formula shows no significant relationship between the differences of $\mu_{\delta}$ and $V$ magnitudes, nor for the color index. Using LSM, a small sinusoidal curve with amplitude $A=0.7 \pm 0.5 \mathrm{mas} \mathrm{yr}^{-1}$ was calculated for ILS and DR2 differences as a function of $\alpha$.

We determined $\mu_{\delta}$ ILS values for 387 stars by calculating corrections of HIPPARCos suitable values. As the input, the ILS latitude data (points with epochs lasting about $80 \mathrm{yr}$ ILS interval) and the HIPPARCos point ( $\delta$ for the epoch 1991.25) were used with suitable weights. Thus meaning, the ILS $\mu_{\delta}$ solution is directly linked to the HIPPARCos positions and proper motions system, but the ILS is independent of the NHIP. Therefore, there is similar behavior for the HIPPARCos and ILS $\mu_{\delta}$ values. Differences between ILS and DR2 are similar to differences between NHIP and DR2. 
Table 5. ILS catalog of $\mu_{\delta}$ for 387 stars.

\begin{tabular}{lll}
\hline \hline HIP number & $\begin{array}{l}\mu_{\delta} \\
{\left[\mathrm{mas} \mathrm{yr}^{-1}\right]}\end{array}$ & $\begin{array}{l}\text { st. dev. of } \mu_{\delta} \\
{\left[\mathrm{mas} \mathrm{yr}^{-1}\right]}\end{array}$ \\
\hline 19 & -15.03 & 0.39 \\
106 & -11.46 & 0.15 \\
$\ldots$ & $\ldots$ & $\ldots$ \\
117622 & -74.80 & 0.57 \\
118224 & -2.75 & 0.08 \\
\hline
\end{tabular}

Notes. Full table is available at the CDS.

The systematic errors of $\mu_{\delta}$ could be larger for double and multiple stars because of the influence of the orbital motion on proper motions. That effect of the orbital motion and proper motions are difficult to separate from each other; especially using short periods of satellite missions, such as HIPPARCOS and Gaia. However, ground-based data, such as ILS data, are of importance. Here, part of the random and systematic errors could be in line with orbital motions of double and multiple stars, and astrometric binaries could be a reason for some discrepancies, which are still unrecognized, between the data from the catalogs. It is necessary to continue investigating astrometric binaries. In the ILS catalog, we found 18 double and multiple stars (using HIPPARCOS and WDS data), but there could be more of them. With the ILS data, we checked over the other three catalogs, and these catalogs are consistent between each other. We hope that these investigations provide more information about the Gaia DR2 data. The presented results are in line with the activity of the IAU Working Group on Astrometry by Small Ground-Based Telescopes. The ILS catalog (see Table 5) is available at the CDS.
Acknowledgements. We thank the referee Dr. van Leeuwen for the very constructive comments, which helped substantially improve the manuscript. This research was supported by the Ministry of Education, Science and Technological Development of the Republic of Serbia (Project No. 176011 "Dynamics and kinematics of celestial bodies and systems"). This work has made use of data from the European Space Agency (ESA) mission Gaia (https://www . cosmos.esa.int/gaia), processed by the Gaia Data Processing and Analysis Consortium (DPAC; https://www.cosmos.esa.int/web/gaia/dpac/ consortium).

\section{References}

Damljanović, G. 2005, Serb. Astron. J., 170, 127

Damljanović, G. 2007, PhD Thesis, University of Belgrade, Serbia

Damljanović, G., \& Pejović, N. 2006, Serb. Astron. J., 173, 95

Damljanović, G., Pejović, N., \& Jovanović, B. 2006, Serb. Astron. J., 172, 41

Eichhorn, H. 1974, Astronomy of Stars Positions (New York: Frederick Ungar Publishing Co.)

ESA 1997, The Hipparcos and Tycho Catalogues, ESA SP-1200 (Noordwijk: ESA Publications Division)

Gaia Collaboration (Prusti, T., et al.) 2016a, A\&A, 595, A1

Gaia Collaboration (Brown, A., et al.) 2016b, A\&A, 595, A2

Ivanov, G., \& Yatsenko, A. 2003, Kinematika i fizika nebesnykh tel, 19, 477

Kovalevsky, J., Lindegren, L., Perryman, M. A. C., et al. 1997, A\&A, 323, 620

Lindegren, L., Lammers, U., Bastian, U., et al. 2016, A\&A, 595, A4

Lindegren, L., Hernández, J., Bombrun, A., et al. 2018, A\&A, 616, A2

Prusti, T. 2012, Astron. Nachr., 333, 453

van Leeuwen, F. 2007, Hipparcos, the New Reduction of the Raw Data (Dordrecht: Springer)

Vondrák, J. 2004, Serb. Astron. J., 168, 1

Vondrák, J., \& Ron, C. 2003, in Journées 2002 - Systèmes de Référence SpatioTemporels, eds. N. Capitaine, \& M. Stavinschi, 49

Vondrák, J., Pešek, I., Ron, C., \& Čepek, A. 1998, Publ. Astron. Inst. Acad. Sci. Czech Republic, 87, 1

Yumi, S., \& Yokoyama, K. 1980, Results of the International Latitude Service in a Homogeneous System 1899.9-1979.0 Central bureau IPMS, Mizusawa 